\title{
ESCRITURA COLABORATIVAEN LÍNEA. UN ESTUDIO PRELIMINAR ORIENTADO AL ANALISIS DEL PROCESO DE CO-AUTORÍA ${ }^{1}$
}

\author{
(ONLINE COLLABORATIVE WRITING, A PRELIMINARY STUDY FOCUSED ON THE ANALYSIS OF \\ THE PROCESS OF CO-WRITING)
}

\author{
Alfonso Bustos Sánchez ${ }^{2}$ \\ Universidad de Barcelona (España)
}

\section{RESUMEN}

Se presenta el análisis exploratorio del seguimiento de una actividad de escritura colaborativa en línea. Desde una perspectiva que integra las ideas de la escritura como una herramienta para aprender y el aprendizaje colaborativo, diseñamos una metodología para el seguimiento del proceso de escritura colaborativa. Se analizaron los resultados de 6 grupos que realizaron un ensayo como producto final en una asignatura de la carrera de Psicología. Los datos preliminares que se presentan en este trabajo permiten identificar grupos cuyo proceso se asocia a procesos de co-autoría real que favorece la aparición de procesos argumentativos y reflexivos y guía el trabajo de grupo hacía procesos cercanos a los esperados en las comunidades de indagación. El trabajo invita a la reflexión sobre uso de los procesos de escritura colaborativa en la enseñanza universitaria para el seguimiento de los estudiantes y la entrega de ayudas durante el proceso.

Palabras clave: escritura colaborativa en línea, co-autoría, educación superior, e-learning, blended-learning, CSCL.

\begin{abstract}
In this article we present an exploratory analysis of online collaborative writing activity. From a perspective that writing is a tool for collaborative learning and teaching, we designed a method to monitor the collaborative writing process. We analyzed the results of six groups whose final assignment in a course for a degree in psychology was to collaborate in the writing of an essay. Preliminary data enabled identification of the groups whose learning could be related to co-writing that favoured the appearance of argumentative and reflexive processes and enabled the group to experience processes
\end{abstract}


related to those expected in communities of inquiry. The article calls for reflection on the use of the processes of collaborative writing in university teaching for monitoring student learning and providing help during the process.

Keywords: online collaborative writing, co-writing, higher education, e-learning, blendedlearning, CSCL.

La educación, en tanto que fenómeno social, no puede mantenerse al margen de la dinámica de cambio que plantean las condiciones sociales, políticas, económicas y de desarrollo que caracterizan a la sociedad del siglo XXI. Dichas condiciones han sido, entre muchos otros factores específicos, las que han favorecido el surgimiento de la cibercultura o la cultura de la sociedad digital. Este término fue acuñado en su momento por Lévy (2007) para referirse a la cultura propia de las sociedades en las que las tecnologías digitales son las formas dominantes para comunicarse, compartir información, conocimiento, investigar, producir, organizarse y administrar. La educación se ve cada vez más inmersa en la dinámica de dicha sociedad hasta el grado de que algunos autores reflexionan, plantean e incluso cuestionan el qué, el cómo y el para qué de la educación del siglo XXI, así como las funciones que les son designadas (Tedesco, 2000). En este contexto, uno de los aspectos más sobresalientes, o que ha generado buena parte de las reflexiones, dudas, e incluso críticas, tiene que ver con la potencialidad para transformar la educación que las tecnologías de la información y la comunicación (TIC) representan en distintos aspectos relativos a la educación inmersa en la "sociedad del aprendizaje" o "sociedad del conocimiento" o "sociedad red” (Castells, 2006; Coll y Martí, 2001).

Desde nuestra perspectiva, la incorporación de las TIC en la educación, cada vez más constante y acelerada, está produciendo una serie de cambios y transformaciones en las formas en que nos representamos y llevamos a cabo los procesos de enseñanza y aprendizaje. En este trabajo nos centramos en el análisis exploratorio del uso de Internet para aprender y enseñar en la universidad, específicamente, en los procesos de uso de los recursos tecnológicos ofrecidos a los estudiantes para desarrollar una tarea de escritura colaborativa. Presentamos los resultados preliminares de una experiencia realizada con estudiantes universitarios y basada en el uso de un conjunto de herramientas tales como foros, Chat y Wiki.

\section{ESCRITURA COMO HERRAMIENTA DEL PENSAMIENTO}

En los últimos años la escritura ha dejado de considerarse desde una perspectiva estática y se ha reconceptualizado hasta dejar de lado su visión de mera trascripción 
de la lengua oral. Además, desde la investigación, se ha puesto mucho más énfasis en su naturaleza procesual que en su carácter de producto terminado y se ha destacado, sobre todo, el papel que tiene respecto del pensamiento. Gracias a ello, entre otras cosas, han surgido una serie de posiciones teóricas al respecto de la escritura y el aprendizaje tales como: el uso epistémico de la escritura, critical writing o escribir para aprender (writing to learn).

Para autores como Olson (1998) es necesario comprender desde una nueva perspectiva el tema de la cultura escrita. El autor destaca, por ejemplo, que desde hace más de tres décadas la lectura y la escritura se reconocen como factores fundamentales para el desarrollo de la racionalidad. Dicho reconocimiento ha permitido que este tópico tenga un sitio relevante en las agendas educativas y de investigación de muchas disciplinas científicas, entre ellas la psicología. Este tópico, además, se ha abordado desde perspectivas centradas en comprender lo que las personas hacen con dichos instrumentos y, sobre todo, desde la necesidad de estudiar y analizar todo lo que está en juego en nuestra alfabetización y en nuestro carácter de alfabetizados.

Desde una perspectiva de uso podemos plantear que la escritura cumple una doble función: la primera, comunicativa, transaccional o interpersonal (escribir para interactuar con otras personas o para comunicarnos); y, la segunda, representativa o ideacional (escribir para expresar conocimientos, ideas, sentimientos, representarnos o recrearnos los objetos de nuestro pensamiento). Desde esta segunda función, la escritura se concibe como un instrumento de toma de conciencia, de autorregulación intelectual o como instrumento para el desarrollo y la construcción del propio pensamiento. Se entiende con ello que la producción escrita, los procesos que el escritor utiliza al componer un texto, favorecen el aprendizaje, el desarrollo del conocimiento sobre sí mismo y sobre la realidad. Para autores como Miras (2000), escribir reflexivamente supone algo más que el dominio de las estrategias de composición, supone la capacidad y la intención de reflexionar y cuestionar los propios conocimientos, la habilidad de identificar los aspectos confusos y contradictorios en el texto, la capacidad de leer de manera estratégica y, sobre todo, la capacidad de releerse.

Para finalizar este apartado conviene señalar, como lo hace Carlino (2005) en su obra "Escribir, leer y aprender en la universidad", que tanto la lectura como la escritura son imprescindibles para desarrollar pensamiento crítico en los estudios universitarios y que ambas habilidades deben desarrollarse también en estos ámbitos y no sólo en la educación básica. Este trabajo, aunque no tiene su foco central en la enseñanza de la escritura académica, sigue el principio propuesto por la autora y se 
propone analizar, de manera exploratoria, los usos que pueden hacer estudiantes universitarios de los recursos tecnológicos que se ponen a su disposición para la regulación de los procesos de escritura colaborativa. En los siguientes apartados presentaremos algunas ideas clave para entender cómo se insertan actualmente las TIC en los procesos de escritura y cómo se ha estudiado la escritura colaborativa.

\section{LAS TIC Y SU RELACIÓN CON LA LECTURA Y LA ESCRITURA}

Antes de presentar algunos análisis que relacionan a la TIC con la escritura y la lectura queremos destacar que las tecnologías de la información y la comunicación se distinguen por tres características principales. La primera, su dimensión simbólica, que hace que se conviertan en potenciales herramientas cognitivas, o mejor aún, en potenciales instrumentos psicológicos dado que también pueden favorecer la regulación de uno mismo y la de los otros (Coll y Marti, 2001). La segunda, las posibilidades que ofrecen para combinar medios y lenguajes - dado su carácter hipermedia-, con lo que se convierten en un recurso mucho más amplio y diverso para comunicar nuestras ideas. La tercera y última, su carácter hipertextual, que abre un horizonte hasta antes insospechado: la escritura puede dejar de ser lineal para expandirse "hipertextualmente" y lograr combinaciones de textos que nos lleven a otros textos o a otras imágenes, audios, videos o cualquier otro recurso que sea necesario dados los propósitos de un texto.

Para Coll (2005), el contexto de la sociedad del siglo XXI, en tanto que sociedad de la información y/o del conocimiento, nos muestra con sobradas evidencias que asistimos a una profunda transfiguración de la lectura, de los procesos y prácticas de lectura y de los conceptos de texto, autor y lector. De acuerdo con el autor, en este contexto, en el que las tecnologías digitales son las responsables de dicha transfiguración, la lectura seguirá siendo el principal instrumento de acceso al conocimiento, acompañada, eso sí, de la exigencia de nuevos saberes, de nuevos conocimientos y habilidades. Al mismo tiempo, las tecnologías también generan nuevas potencialidades para crear y acceder a otros tipos de textos y a nuevos tipos de prácticas letradas, en suma, nuevas modalidades de producción, transmisión, acceso y uso de los textos escritos (Millán, 2000. Cit. en: Coll, 2005). En este sentido, Coll (2005, p. 6) propone, siguiendo a Chartier (2000), que la transfiguración supone un cambio epistemológico fundamental en dos vertientes: la del autor y la del lector. Desde el punto de vista de quien escribe supone formas de organización textual que superan la lógica lineal y deductiva de los textos en papel para dar paso a la posibilidad de arreglos no lineales, abiertos y relacionales -lo que supondría nuevas formas de 
argumentación y construcción de sentido-. Desde el punto de vista del lector, se le ofrece la oportunidad de una exploración mucho más abierta y a voluntad que, gracias a los hipervínculos, permite una relación mucho más significativa entre las partes que componen el texto y sus características hipermedia (sonidos, imágenes u otros recursos extra-textuales). Dicha posibilidad de "recomponer" el texto permitiría al lector, por ejemplo, la construcción de significados no previstos por el autor.

Desde ambas vertientes, la del escritor y la del lector, aparecen, además de las potencialidades señaladas, una serie de desventajas relativas sobre todo a la estructura no lineal del texto y la compleja red de informaciones interrelacionadas que podrían suponer los textos electrónicos. Por un lado, pueden llevar al autor a crear textos interminables, confusos e incluso desestructurados; y por otro, llevar al lector a un mar de información innavegable si no se cuenta con los nuevos saberes, conocimientos y habilidades que el texto electrónico puede demandar (dichas habilidades han sido agrupadas por algunos autores bajo las nociones de alfabetización digital -digital literacy- o multilfabetización -multiliteracies-).

\section{LA ESCRITURA COLABORATIVA SOPORTADA POR ORDENADORES}

Desde las corrientes más actuales al respecto del aprendizaje se plantea, cada vez más, la potencia de aprender con los otros y de los otros, de buscar la forma en que los estudiantes, en todos los niveles educativos, cuenten con oportunidades de aprender en grupo colaborando con sus pares para resolver problemas auténticos que supongan un uso situado del conocimiento. Además se privilegian las perspectivas que permiten aprender a colaborar, es decir, a escuchar las opiniones de los otros, considerarlas, buscar acuerdos e instrumentar juntos las soluciones generadas por el grupo (Edwards y Mercer, 1988; Wertsch, 1988; Coll, 2001; Mercer, 2001). En la sociedad del siglo XXI, se valora positivamente cualquier iniciativa de gestionar oportunidades para el aprendizaje colaborativo.

Por un lado, este contexto, el de la alta valoración del aprendizaje colaborativo, se ha visto nutrido por las sólidas aproximaciones al estudio de lo que se conoció primero como trabajo colaborativo mediado por computadoras (CSCW, por sus siglas en inglés), y que se ha desarrollado después hacía la aproximación del aprendizaje colaborativo mediado por ordenadores (CSCL, por sus siglas en inglés). Este paradigma en emergencia se centra en analizar y comprender cómo la colaboración y la tecnología pueden favorecer la construcción del conocimiento y cómo el aprendizaje colaborativo mediado por los ordenadores puede mejorar la interacción y el trabajo 
en grupo (Schmidt, 2001; Scardamalia y Bereiter, 2003). Por otro lado, desde una visión constructivista y sociocultural de los procesos de enseñanza y aprendizaje se pone el acento en el seguimiento que los profesores, y los mismo participantes, pueden hacer del proceso colaborativo y la entrega de ayudas ajustadas y diversas gracias a que, ciertos usos de la tecnología, permiten transparentar aspectos de la colaboración relativos a quién, qué, cómo y cuándo aportan para la consecución de la tarea (Coll, Mauri y Onrubia, 2006).

Ahora bien, gracias al desarrollo de las propuestas teóricas para aproximarnos al estudio del aprendizaje con los otros, se han desarrollado aproximaciones teóricas y aplicadas relativas a la escritura colaborativa que toman cada vez más relevancia en el contexto al que nos estamos refiriendo. Storch (2005) hace un excelente recuento de las aproximaciones que se han desarrollado respecto de la escritura colaborativa en el salón de clases. La autora plantea por ejemplo que, en las primeras aproximaciones, las posibilidades del trabajo en grupo o entre pares para escribir se limitaban o al proceso de generación de ideas (brainstormig) o al proceso final de revisión (peer review). Destaca, además, que algunas investigaciones mostraban que, sobre todo el proceso de revisión entre pares, generaba beneficios para los participantes relativos a, por una parte, su capacidad de considerar las posiciones de los otros (Ferris, 2003; Cit. En: Storch, 2005); y, por otra, en el desarrollo de habilidades tanto para la escritura como para la lectura analítica y crítica (Nystrand y Brabdt, 1989; Cit. en: Storch, 2005). Sin embargo, la autora plantea también que la tendencia a potenciar y estudiar la escritura colaborativa sólo como proceso de revisión entre pares supone una deficiencia en la aproximación dado que se centra en el producto y no considera el proceso de escritura. Investigaciones más recientes demuestran que, respecto del proceso para la escritura colaborativa, los estudiantes deberían participar a lo largo de todo el proceso de escritura, lo que significaría que los participantes en dicho proceso deberían compartir la responsabilidad en la producción de un texto tanto a nivel de estructura, de contenido y de lenguaje. Las ventajas de una posición de coautoría, más que de revisión entre pares, se centran por ejemplo en aspectos como los siguientes: (i) se favorece el pensamiento reflexivo (sobre todo si los participantes ponen en marchan mecanismos para defender o explicar mejor sus ideas); (ii) los participantes pueden rebasar los niveles centrados en la ortografía o la gramática para abordar cuestiones relativas al discurso; por último, (iii) puede favorecer el conocimiento que los participantes desarrollan sobre el lenguaje (Keys, 1994; Storch, 2002; Storch, 2003). Finalmente, el debate al respecto del estudio de la escritura colaborativa se centra en la necesidad de investigar tanto los productos como los procesos que los estudiantes ponen en marcha cuando escriben colaborativamente, así como las expectativas, actitudes y reflexiones que pueden tener respecto de dicho proceso. 


\section{A. Bustos \\ Escritura Colaborativa en Línea. Un Estudio Preliminar Orientado al Analisis del Proceso ...}

De acuerdo con Kraut, Galegher, Fish, y Chalfonte (1992; citados en: Barile y Durso, 2002) la escritura colaborativa puede equiparse a la escritura individual en varios sentidos: primero, el proceso de escritura grupal puede equipararse al proceso individual en términos de macro-estructura o de la estructura formal del texto narrativo. Segundo, ambas siguen, generalmente, tres fases que pueden intercalarse y que aparecen de manera recursiva: planificación, escritura y revisión (Hayes y Flower, 1986). Tercero, ambas tareas son de carácter abierto, es decir, no hay ni una definición única ni un procedimiento igual para completarla, el procedimiento está abierto para cambios en cualquier momento tanto en la dirección como en el foco.

No obstante, la escritura colaborativa presenta diferencias sustanciales respecto de la escritura individual. Por una parte, y de acuerdo con Rada y Wang (1997), la diferencia más relevante se puede identificar en los requerimientos de comunicación y coordinación que supone el trabajo de grupo. Para Barile y Durso (2002) esto supone un rasgo especial en la escritura colaborativa que definen como "dependiente de la comunicación” y de ahí que señalen la necesidad de ofrecer a los grupos de trabajo los instrumentos más adecuados para que desarrollen los más eficientes estilos de comunicación. Estos autores sostienen que la variación de los estilos de comunicación soportados por diferentes recursos tecnológicos puede impactar en la efectividad del grupo. Por otra parte, la escritura colaborativa requiere también de un aumento en la posibilidad de interactividad y expresividad. De acuerdo con Galegher y Kraut (1996), en el contexto de la escritura grupal, la interactividad se entiende como la capacidad para mantener intercambios rápidos de información junto con la habilidad para responder cuanto antes a la información del otro participante, mientras que la expresividad se refiere a la habilidad para comunicar una idea.

Con base en estas similitudes y diferencias, Barile y Durso (2002) plantean que la escritura colaborativa es una actividad multitarea que involucra un proceso de lluvia de ideas y otro de búsqueda de consenso que, como ya planteábamos, pueden darse de manera recursiva e intercalada. Los autores caracterizan el proceso como sigue: una vez que las ideas se han propuesto, el grupo debe decidir qué ideas selecciona para trabajarlas, cómo estructurar el documento, qué contenidos deberían incluir y qué revisiones deberían hacer antes de llegar a un texto final. En su opinión, cuando este proceso se plantea mediado por ordenador requiere, cuando menos, recursos que permitan suficientes niveles de interactividad y expresividad.

Erkens, Jaspers, Prangsma y Kanselaar (2005) sugieren que la principal ventaja de la escritura colaborativa, comparada con la individual, es la posibilidad de recibir y ofrecer retroalimentación inmediata gracias a su característica como tarea 
"argumentativa". En este sentido, Stein, Bernas y Calicchia (1997) destacan que el carácter argumentativo de la tarea favorece el aprendizaje ya que los miembros de un grupo se consideran unos a otros fuentes de conocimiento, además que los participantes negocian entre ellos los propósitos, planes, conceptos y dudas. Los autores agregan que en la escritura colaborativa los participantes deben llegar a acuerdos respecto del contenido y la organización del texto, eso supone la necesidad de estar coordinados y discutir.

Respecto de la escritura colaborativa soportada por ordenadores o en línea, Erkens et al. (op.cit), siguiendo estos principios, desarrollaron un entorno digital para el trabajo en grupo con el objeto de apoyar a los estudiantes durante la fase de planeación ofreciendo herramientas específicas para el trabajo con los conceptos relativas a la planeación, la organización y la estructuración. Por su parte, Barile y Durso (2002) investigaron si el uso de sistemas de comunicación mediada por ordenadores (CMC, por sus siglas en inglés) durante una tarea de escritura grupal era un modo efectivo de comunicación. Compararon tres condiciones: cara a cara, comunicación síncrona mediada por ordenador (Netmeeting) y correo electrónico. Los resultados sugieren que el correo electrónico solo no es apropiado para la escritura colaborativa, mientras que el sistema síncrono de comunicación, como netmeeting, podría ser viable para obtener resultados positivos en el trabajo en grupo.

Para concluir este apartado, debemos tener presente que, junto con el desarrollo de las tecnologías para leer y escribir, es fundamental considerar el desarrollo de propuestas tecnopedagógicas para aprender escribiendo con los otros o para aprender solucionando problemas con los otros a través de la revisión entre pares en línea o de la escritura conjunta en línea (On-line Collaborative Writing) ${ }^{3}$. De entre las tecnologías para el soporte de los procesos de escritura en línea podemos destacar los siguientes: (i) la tecnología Wiki que se pude categorizar, según Piscitelli (2005, p.87) como una "aplicación de informática colaborativa". Dicha tecnología suele definirse como una herramienta efectiva para la escritura colaborativa. Permite crear colectivamente documentos web usando un simple esquema de etiquetas y marcas y no requiere de una instancia jerárquica que apruebe la publicación de las modificaciones. (ii) Los “Entornos para la construcción del conocimiento" (Knowledge Building Environment). Dichos entornos parten del supuesto teórico propuesto por Scardamalia y Bereiter (2003) quienes, desde una perspectiva constructivista, plantean que la construcción del conocimiento es un proceso de creación de "nuevos artefactos cognitivos" como resultado de tener objetivos comunes, de la discusión grupal y de la síntesis de las ideas. La construcción del conocimiento implica la acción colectiva de indagación respecto de un tópico específico para el avance en la 
comprensión gracias al cuestionamiento interactivo, el diálogo y la mejora continua de las ideas. Las ideas son, por lo tanto, el medio de operación en un entorno para la construcción del conocimiento. Los participantes asumen el control en su propio proceso de construcción de conocimiento incluyendo aspectos relativos a la planeación, la ejecución y la evaluación.

Hasta aquí, nuestra aproximación inicial al campo de estudio que supone la escritura colaborativa mediada por las TIC. Dicho campo comparte, desde nuestra perspectiva, los mismos aspectos que ya citamos como relevantes para el estudio de la escritura colaborativa en el salón de clases: la necesidad de investigar tanto los productos como el proceso, las expectativas, actitudes y reflexiones de los estudiantes. Integra, además, la necesidad de identificar y analizar algunas de las ventajas o limitaciones que supone para el proceso la mediación llevada a cabo por recursos tecnológicos, específicamente, por recursos en línea o virtuales.

Como habíamos anunciado, este trabajo se presenta como un primer acercamiento a la investigación de la escritura colaborativa en línea (On-line Collaborative Writing). Nuestra investigación plantea un seguimiento pormenorizado de los usos que hacen los participantes en grupos de trabajo colaborativo, que tienen como tarea la escritura conjunta de un texto en línea, de los cuatro tipos de recursos puestos a su disposición: acceso a materiales de lectura y apoyo, un foro de comunicación asíncrona, un sistema de conferencia por ordenador o Chat y un sistema de escritura colaborativa en línea basado en tecnología Wiki. El objetivo del presente trabajo es identificar, desde una perspectiva exploratoria inicial, procesos de uso de los recursos para la escritura colaborativa en línea que permitan suponer altos niveles de coautoría o coordinación mutua en el logro de una tarea de escritura conjunta.

\section{METODOLOGÍA}

El estudio se desarrolló en el marco del proyecto general "Aportaciones de la interactividad en línea para la gestión de la formación universitaria” que se realiza en el proyecto de Investigación Psicoeducativa de la Universidad Nacional Autónoma de México Campus Iztacala. Este trabajo se refiere a los datos obtenidos durante el semestre 2006-2 con estudiantes de mitad de la licenciatura en Psicología de la asignatura Desarrollo y Educación Teoría I. La asignatura se imparte, desde hace más de dos años, bajo un modelo bimodal (blended-learning) que combina clases presenciales con clases en línea utilizando múltiples combinaciones de recursos y actividades que ofrece Moodle (un entorno virtual para el aprendizaje que permite 
trabajar con foros, chat, wikis y que facilita, además, la creación de grupos de trabajo). El arreglo del entorno utilizado en nuestra modalidad tipo blended-learning sigue algunos de los principios sugeridos en los trabajos de Onrubia, Bustos, Engel y Segués (2006) y de Onrubia, Coll, Bustos y Engel (2006) para el uso de las herramientas de comunicación y trabajo colaborativo tanto en gran grupo como en pequeño grupo.

A lo largo de la asignatura referida se desarrollaron tres modalidades de trabajo en línea: la modalidad síncrona (Chat), la modalidad asíncrona basada en foros de discusión y la modalidad de ensayo colaborativo en línea. La modalidad a la que refiere este estudio es la modalidad de ensayo colaborativo. Dicha modalidad se llevó a cabo al final de la asignatura y no tuvo un carácter acreditativo definitorio, aunque sí se evaluó para asignar a los estudiantes un extra en su calificación final.

La tarea se centró en la revisión y discusión de un caso, la revisión de las lecturas obligatorias y optativas relativas a los contenidos y la elaboración del ensayo colaborativo mediante un wiki. Las características del ensayo se definieron como sigue: "el ensayo no debería rebasar 10 folios ni tampoco sería de menos de 5 . Debería contener la siguiente estructura: introducción, marco teórico, ejemplos prácticos y conclusiones". Finalmente, se plantearon una serie de pautas y fases para el proceso de realización del ensayo asociadas a las 3 semanas de trabajo y los tres capítulos teóricos a revisar. Primera semana: análisis conjunto del caso y discusión en grupo sobre sus características (capítulo 10. Planeación, instrucción y tecnología). Segunda semana: discusión en grupo sobre las características del ensayo (capítulo 11. Cómo motivar a los alumnos a aprender); tercera semana: discusión en grupo sobre las características del ensayo (capítulo 12. Manejo del aula). Además, se colocaron en la plataforma un manual y un tutorial en texto y video sobre el uso y funcionamiento del Wiki, la forma de trabajo, la descripción del caso y los materiales complementarios (lectura reforma, tarjeta guía reforma).

Participaron en la experiencia 29 estudiantes (20 mujeres y 9 hombres). Los estudiantes conformaron, de acuerdo a sus propios criterios y con la única consigna de no ser más de 4 integrantes, 11 equipos de trabajo colaborativo (7 equipos de 3 integrantes y 4 equipos de 2). Los datos analizados en este trabajo se refieren sólo a 6 grupos de trabajo integrados por 16 estudiantes (14 mujeres y 2 hombres).

A partir del inicio de la modalidad colaborativa todo el proceso de trabajo se realizó completamente en línea. Se recogieron datos electrónicos relativos al acceso y uso de la herramienta por cada miembro del equipo colaborativo y se registraron aspectos como lectura de documentos previos al inicio del trabajo colaborativo, 
entrega de resumen de lecturas obligatorias, seguimiento y uso del foro avisos, seguimiento y uso del foro de pequeño grupo así como un detallado resumen del trabajo en el editor colaborativo. Además, se recogieron todas las versiones de los textos colaborativos.

\section{RESULTADOS}

En este trabajo, que ya definíamos como de carácter exploratorio, no entraremos aún en el proceso de análisis cualitativo y cuantitativo de los textos resultado del trabajo colaborativo, pero anticipamos que sí es una de las fases siguientes en nuestro proceso de investigación. Nos centraremos, por lo tanto, en el análisis cuantitativo y descriptivo de los datos que nos permiten hacer un seguimiento pormenorizado del proceso respecto de varios aspectos.

Hemos agrupados los datos electrónicos relativos al acceso y uso de la herramienta por cada miembro de los equipos colaborativos en los siguientes apartados y subapartados:

- Lectura de documentos previos al inicio del trabajo colaborativo:

- Forma de trabajo, lectura reforma, tarjeta guía reforma, lectura caso, tutorial Wiki video y tutorial Wiki texto);

- Entrega de resumen de lecturas obligatorias:

- De la lectura 1 a la lectura 6;

- Seguimiento y uso del foro avisos -general-, así como del foro y chat de pequeño grupo;

- Trabajo en el editor colaborativo:

- Días de revisión wiki, días con edición del wiki, temas wiki editados, número de ediciones y número de versiones creadas o editadas por apartado del texto.

Respecto del apartado 1 (lectura de documentos previos al inicio del trabajo colaborativo), encontramos que el $94 \%$ de los participantes revisaron la forma de trabajo, el $88 \%$ el tutorial wiki video, el $81 \%$ revisaron la lectura reforma y su correspondiente tarjeta guía y, finalmente, sólo el $75 \%$ revisaron el tutorial wiki 
texto. Cabe señalar que el promedio de revisiones de documentos fue de 10,06 lo que nos indica el hecho de que los participantes revisaron en línea más de una vez alguno de los documentos (5 como valor máximo y o como mínimo). $\mathrm{Al}$ menos dos estudiantes (MD del grupo 10 e I del grupo 11) no revisaron, respectivamente, la forma de trabajo y ninguno de los tutoriales del wiki. Ambos estudiantes, como veremos posteriormente, también son los estudiantes con menos contribuciones al proceso de trabajo colaborativo.

Si revisamos los datos de los grupos, encontramos que para el grupo 3 todos los estudiantes revisaron todos los documentos. Una de las participantes del grupo 1 revisó todos los documentos (RI) mientras que sus compañeros revisaron todos excepto el tutorial Wiki texto. En el grupo 5, todas revisaron todo (excepto BM que no leyó la lectura complementaria). En el grupo 7, solamente la participante MdeA leyó todo, T no revisó ni la lectura complementaria ni la tarjeta guía de esa misma lectura. En el grupo 10, HL leyó todos los recursos, MdeC revisó todos los materiales excepto el tutorial wiki texto mientras que MD no revisó la forma de trabajo y tampoco la tarjeta guía de la lectura complementaria ni el tutorial del wiki video. Finalmente, en el grupo 11, la participante A leyó todo mientras que la participante FdeJ no revisó la lectura reforma, por su parte la participante I no revisó ninguno de los tutoriales wiki.

Hasta aquí, los datos relativos al seguimiento de la forma en que los integrantes de los equipos consultaron o no los materiales relativos al inicio de la actividad nos dan una primera idea del contexto que los participantes podían compartir, al menos, respecto de la representación de la tarea y de las herramientas que utilizarían para tal fin. Consideramos que estos datos son relevantes en tanto que se refieren al hecho de compartir las reglas de participación y de la tarea así como las características de las herramientas con las que se trabajará. Cabe señalar que en todos los grupos, excepto el grupo 10, cabría esperar que todos los participantes compartieran cierta claridad respecto de la forma de trabajo, en tanto que todos habían consultado el documento descriptivo de la actividad al menos una vez. De la misma forma, en todos los grupos, excepto en el grupo 11, cabría esperar que todos compartieran una representación más o menos clara del recurso electrónico a utilizar, dado que los participantes habían consultado al menos alguno de los tutoriales.

Respecto del apartado 2 (entrega de resumen de lecturas obligatorias), los datos nos indican que el $63 \%$ del total de participantes entregaron resumen de la lectura 1 , el 69\% de la lectura 2, el 75\% de la lectura 3, el 64\% de la lectura 4, sólo el 56\% de la lectura 5 y, por último, el $75 \%$ de la lectura 6 . El promedio de resúmenes de lectura 
entregados por todos los participantes fue de 4,06 (con un valor máximo de 6 -lo esperado-, y hasta un valor mínimo de 0). Al menos 2 participantes entregaron los 6 resúmenes esperados; 10 entregaron entre 5 y 3 resúmenes y 3 entregaron sólo entre 1, 2 o ninguno de los resúmenes esperados. Al revisar los datos a lo interno de los grupos tenemos que entre los tres integrantes del grupo 1 entregaron el 94\% de los resúmenes esperados (muy por encima del promedio general del grupo clase), sólo el participante RI no entregó el resumen correspondiente a la primera lectura. En el grupo 3 ambos integrantes entregaron 5 resúmenes de los 6 esperados. El grupo 5, como grupo, sólo entregó la mitad de los resúmenes esperados (el participante D entregó 5, el participante CE 3 y el participante BM ninguno). Los dos participantes del grupo 7 entregaron 9 lecturas de 12 (la participante T entregó 4 y la participante MdeA 5). El grupo 10 fue el otro grupo que, como grupo, sólo entrego la mitad de los resúmenes de lectura (las participantes HL y MdelC entregaron 5 y 4 resúmenes, respectivamente, y el participante MD sólo entregó un resumen de 6 esperados). Por último, en el grupo 11 las participantes A e I entregaron 5 y 4 resúmenes respectivamente, mientras que la participante FdeJ entregó sólo 2.

Hasta aquí, los datos relativos a la entrega de resúmenes de las lecturas obligatorias se relacionan con lo que Erkens, et al. (2005) refieren como el papel de los estudiantes para cuidar la coherencia o consistencia de la base de conocimiento colectivo. Podemos identificar algunos aspectos relativos a, por un lado, el compromiso dispar que los miembros de los equipos parecen tener respecto del trabajo personal con los contenidos para el abordaje de la tarea escrita (dado que en la mayoría de equipos unos cuantos leen todo o casi todo y el resto lee muy poco o casi nada). Lo esperado sería que, como en el caso de los equipos 1 y 7 , todos los miembros del equipo entregaran el resumen solicitado de los contenidos, por dos razones: primero, porque la tarea de escribir un ensayo colaborativo exige que, además de compartir una representación ajustada de la tarea en sí misma (ver el apartado anterior), se comparta la base de conocimientos de partida sugerida para el abordaje de la tarea de escritura; y, segundo, porque los resúmenes de las lecturas podrían ser considerados como muy buenos "cimientos" para procesos como la puesta en común de ideas, la discusión, la negociación y, sobre todo, la posibilidad de iniciar aportaciones al texto colaborativo en la herramienta Wiki. Desde nuestra perspectiva, el trabajo con los contenidos propuestos, así como el proceso personal de enfrentarse a la lectura y elaboración del resumen como mecanismos para comprender el caso al que se enfrentarían debería ser una fase básica o determinante de lo que algunos autores llaman la base compartida de conocimientos, sin la cuál, difícilmente, se pueden echar a andar verdaderos procesos de colaboración, sobre todo, si hablamos de escritura colaborativa. 
Respecto del apartado 3 (seguimiento y uso del foro avisos -general- así como del foro y chat de pequeño grupo), nos centramos en el uso del foro y chat de pequeño grupo como recursos de los grupos para comunicarse, gestionar su proceso y tomar decisiones. Los datos que aquí presentamos se refieren a lo que autores que presentamos en nuestra revisión refieren como argumentación, toma de decisiones, discusión, presentación de ideas, etc.

Encontramos que, respecto del foro de pequeño grupo, los grupos 3,7 y 11 no utilizaron esta herramienta para comunicarse entre ellos (en los grupos 7 y 11 una de las participantes realizó una aportación en el foro que no recibió respuesta alguna). Los grupos 1, 5 y 10 sí utilizaron el foro para comunicarse durante su proceso de trabajo. Como puede observarse en la tabla I, los grupos 1 y 5 realizaron hasta 9 aportaciones, mientras que el grupo 10 sólo realizó 4 aportaciones (éste fue el único grupo en el que uno de los participantes no aportó nada al foro-MD-). En los otros dos grupos las aportaciones se distribuyen entre los 3 participantes del grupo. Finalmente se puede observar que el grupo 1 realizó un acceso más compartido y un número de aportaciones más homogéneo -cabría esperar un trabajo mejor distribuido-. En el grupo 5 encontramos aportaciones homogéneas en el grupo, pero un seguimiento más constante sólo del participante $\mathrm{CE}$ que, por otra parte, es también el que más escribe. Por último, en el grupo 10 es claro que el proceso de discusión o acuerdos en el foro se centra en dos de las participantes, con mucho más actividad por parte de la participante HL -que es quien entró más al foro y quien escribió más-.

\begin{tabular}{|c|c|c|c|c|}
\hline & $\begin{array}{c}\text { Total de aportaciones } \\
\text { grupales }\end{array}$ & Estudiante & $\begin{array}{c}\text { Número de } \\
\text { aportaciones }\end{array}$ & $\begin{array}{c}\text { Días de revisión } \\
\text { del foro }\end{array}$ \\
\hline \multirow{3}{*}{ Grupo 1} & \multirow{3}{*}{9} & $\mathrm{EQ}$ & 3 & 10 \\
\hline & & $A$ & 3 & 7 \\
\hline & & $\mathrm{RI}$ & 3 & 8 \\
\hline \multirow{3}{*}{ Grupo 2} & \multirow{3}{*}{9} & $\mathrm{D}$ & 3 & 4 \\
\hline & & CE & 4 & 11 \\
\hline & & BM & 2 & 3 \\
\hline \multirow{3}{*}{ Grupo 3} & \multirow{3}{*}{4} & $\mathrm{HL}$ & 3 & 12 \\
\hline & & $\mathrm{MdC}$ & 1 & 9 \\
\hline & & $\mathrm{MD}$ & 0 & 2 \\
\hline
\end{tabular}

Tabla 1. Frecuencias de aportaciones y de revisión en el foro de pequeño grupo

Con respecto al Chat de pequeño grupo, encontramos que, como puede observarse en la tabla II, sólo los grupos 1, 7, 10 y 11 realizaron alguna sesión con este recurso durante la realización de su ensayo. Los grupos 1 y 10, que también 
utilizaron el foro de pequeño grupo para comunicarse, sólo realizaron una sesión, mientras que los grupos 7 y 11, que no utilizaron el foro, realizaron dos sesiones de Chat. Es importante destacar aquí que el grupo 3 fue el único grupo que no utilizó ni el foro ni el chat para comunicarse.

\begin{tabular}{|c|c|c|c|}
\hline & $\begin{array}{l}\text { Total de sesiones de } \\
\text { Chat abiertas }\end{array}$ & Estudiante & $\begin{array}{l}\text { Número de } \\
\text { aportaciones }\end{array}$ \\
\hline \multirow{3}{*}{ Grupo 1} & \multirow{3}{*}{1} & $E Q$ & 55 \\
\hline & & $A$ & 25 \\
\hline & & $\mathrm{RI}$ & 32 \\
\hline \multirow{2}{*}{ Grupo 7} & \multirow{2}{*}{2} & $\mathrm{~T}$ & 61 \\
\hline & & $\mathrm{MdA}$ & 71 \\
\hline \multirow{3}{*}{ Grupo 10} & \multirow{3}{*}{1} & $\mathrm{HL}$ & 13 \\
\hline & & $\mathrm{MdC}$ & 50 \\
\hline & & $\mathrm{MD}$ & 41 \\
\hline \multirow{3}{*}{ Grupo 11} & \multirow{3}{*}{2} & AV & 74 \\
\hline & & Fdj & 112 \\
\hline & & 1 & 109 \\
\hline
\end{tabular}

Tabla 2. Sesiones y aportaciones al Chat de pequeño grupo

Finalmente, respecto del apartado 4 (trabajo en el editor colaborativo) presentamos, primero, los datos agrupados relativos a la gestión del tiempo (entendido como los días de la actividad que se dedicaron a revisar o editar el texto colaborativo). Como se puede apreciar en la tabla III, los grupos 3, 5 y 11 fueron los tres grupos con mucha menor actividad. Sólo dedicaron entre 1 y 2 días para la edición de su texto colaborativo (normalmente los últimos días), esto puede ser entendido como indicador de dos tipos de acciones de los grupos: una, la muy poco estratégica acción de dejar la tarea de escribir hasta el último momento (por ello hacen todo en un solo día) o, dos, la acción de realizar la tarea off-line (presencial) y responsabilizar a uno de los participantes para entregarla on-line (en línea) el último día. Esta última acción es muy clara en el grupo 5 dado que sólo una de las integrantes dedicó un día a la edición. En el grupo 11, aún cuando sólo dedicaron un día, todos los miembros participaron de la edición. Mientras que en el grupo 3 la participante EF dedicó dos días a editar su texto y la participante KE sólo 1.

Por otra parte, los grupos 1, 7 y 10 fueron los grupos con mayor actividad. Con excepción del participante MD del grupo 10, en estos grupos dedicaron entre 5 y 10 días para la revisión de los textos y entre 2 y 4 días para la edición. Por otra parte, al contrario que los grupos 3, 5 y 11, en estos grupos todos los participantes dedicaron 
más de un día para la edición de los apartados de su ensayo. En este sentido es relevante destacar dos acciones, que sí pueden ser calificadas como estratégicas, en estos grupos: (i) dedicaron al menos entre 2 y 4 días a la edición y, (ii) en todos los grupos concurrieron todos los participantes, dedicando más de dos días a la edición.

\begin{tabular}{|c|c|c|c|}
\hline \multirow{4}{*}{ Grupo 1 } & Estudiante & $\begin{array}{c}\text { Días de revisión } \\
\text { del texto } \\
\text { colaborativo }\end{array}$ & $\begin{array}{c}\text { Días de edición } \\
\text { del texto } \\
\text { colaborativo }\end{array}$ \\
\cline { 2 - 4 } & $\mathrm{EQ}$ & 10 & 4 \\
\hline \multirow{3}{*}{ Grupo 3 } & $\mathrm{A}$ & 9 & 2 \\
\hline \multirow{3}{*}{ Grupo 5 } & $\mathrm{RI}$ & 7 & 4 \\
\cline { 2 - 4 } & $\mathrm{KE}$ & 2 & 2 \\
\hline \multirow{3}{*}{ Grupo 7 } & $\mathrm{D}$ & 6 & 1 \\
\cline { 2 - 4 } & $\mathrm{CE}$ & 3 & 0 \\
\hline \multirow{3}{*}{ Grupo 10 } & $\mathrm{BM}$ & 2 & 0 \\
\cline { 2 - 4 } & $\mathrm{TdA}$ & 4 & 1 \\
\cline { 2 - 4 } & $\mathrm{HL}$ & 5 & 4 \\
\hline \multirow{3}{*}{ Grupo 11 } & $\mathrm{MdC}$ & 8 & 3 \\
\cline { 2 - 4 } & $\mathrm{MD}$ & 8 & 3 \\
\cline { 2 - 4 } & $\mathrm{AV}$ & 1 & 1 \\
\hline \multirow{2}{*}{} & $\mathrm{FdJ}$ & 8 & 1 \\
\hline
\end{tabular}

Tabla 3. Días dedicados al proceso de revisión y edición del documento Wiki (por grupo y participantes)

Continuamos con los datos relativos al apartado 4 (trabajo en el editor colaborativo) y presentamos, enseguida, los datos agrupados respecto de la gestión del texto y sus apartados (entendido como el número de temas editados por los miembros de los grupos y el número de ediciones totales).

Como cabía esperar, los grupos 3, 5 y 11 (ver tabla 4), que mostraron las acciones menos estratégicas en la gestión del tiempo para escribir, así como en la participación de todos los miembros del grupo, dado que mostraron un proceso de escritura caracterizado por las siguientes acciones: primero, la edición de un solo tema (para el caso del grupo 3) en el que se incluyó todo el conjunto del texto, y un número de ediciones muy bajo (sólo 3 ediciones entre las dos participantes miembros de este equipo). Segundo, la edición de los 4 temas correspondientes al texto, pero 
realizados por solo un participante y con un número de versiones muy bajo (6). Este fue el caso del grupo 7 cuyo texto sólo fue editado por la participante BM. Tercero y último, la edición de los 4 apartados del texto con variación de participación de todos los miembros del grupo, tanto respecto del tema en el que se participa como de las ediciones realizadas a cada tema. Tal fue el caso del grupo 11, en el que una de las participantes (FdI) edita los 4 temas del ensayo (con un alto número de ediciones -14), otra de ellas (I) edita al menos 3 temas con una frecuencia de edición muy baja (3 ediciones) y, finalmente, la última de ellas (AV) sólo edita un tema con hasta 2 ediciones.

Conviene señalar que el proceso de escritura de estos grupos se caracteriza, por un lado, por la falta de seguimiento de las indicaciones para la tarea (dado que se centran en estrategias de copiar y pegar y en la participación de solo un miembro del grupo como gestor de la tarea de trasladar lo hecho en otros espacios al Wiki). Por otro lado, se caracteriza por utilizar una estrategia acumulativa para la constitución de su texto con escasa o ninguna dedicación para la corrección, ampliación, argumentación o mejora de las ideas. Cabe señalar que estos grupos no siguieron la indicación que se señalaba en la forma de trabajo relativa a que cada estudiante debería hacer sus propias aportaciones al texto colaborativo y deberían hacer alguna aportación al menos 3 ocasiones durante cada semana.

Como también cabía esperar después de analizar la gestión del tiempo de escritura, podemos observar que los grupos 1, 7 y 10 (ver tabla IV) abordaron la tarea desde una perspectiva mucho más estratégica caracterizada por otra serie de acciones. Primero, en todos los grupos se observa la participación de todos los miembros del grupo para la edición de todos los temas del texto (sólo en algún caso algún miembro de los grupos 1 y 7 edita sólo 3 de 4 temas). Segundo, en estos grupos se observa un alto número de ediciones del texto (el promedio de ediciones para los tres grupos está entre 11 y 12 ediciones, mientras que el promedio más alto de los grupos anteriores fue de 6). Sin embargo, cabe señalar que para el grupo 1 destaca el hecho de que sigue siendo una de las participantes (A) quien realiza muchas de las tareas de edición del texto -casi la mitad de la ediciones del texto son realizadas por ella-, mientras que en los otros dos grupos la frecuencia de ediciones se distribuye mucho más equitativamente. Lo más destacable de estos datos es que todos participan en todos los temas y que las acciones no se limitan a acumular sino que se complementan con correcciones, ampliaciones, supresiones e incluso cambios en la estructura en la que se presenta la información. 


\begin{tabular}{|c|c|c|c|}
\hline & Estudiante & $\begin{array}{c}\text { Temas Wiki } \\
\text { editados }\end{array}$ & $\begin{array}{c}\text { Número de } \\
\text { ediciones }\end{array}$ \\
\hline \multirow{3}{*}{ Grupo 1 } & $\mathrm{EQ}$ & 4 & 9 \\
\cline { 2 - 4 } & $\mathrm{A}$ & 4 & 17 \\
\cline { 2 - 4 } Grupo 3 & $\mathrm{Rl}$ & 3 & 11 \\
\cline { 2 - 4 } & $\mathrm{EF}$ & 1 & 2 \\
\hline \multirow{3}{*}{ Grupo 5 } & $\mathrm{KE}$ & 1 & 1 \\
\cline { 2 - 4 } & $\mathrm{D}$ & 0 & 0 \\
\hline \multirow{3}{*}{ Grupo 7 } & $\mathrm{CE}$ & 0 & 0 \\
\cline { 2 - 4 } & $\mathrm{M}$ & 4 & 6 \\
\hline \multirow{3}{*}{ Grupo 10 } & $\mathrm{MdA}$ & 3 & 11 \\
\cline { 2 - 4 } & $\mathrm{HL}$ & 4 & 11 \\
\cline { 2 - 4 } & $\mathrm{MdC}$ & 4 & 14 \\
\hline \multirow{3}{*}{ Grupo 11 } & $\mathrm{MD}$ & 4 & 2 \\
\cline { 2 - 4 } & $\mathrm{AV}$ & 0 & 14 \\
\cline { 2 - 4 } & $\mathrm{FdJ}$ & 1 & 3 \\
\hline
\end{tabular}

Tabla 4. Proceso de edición del documento Wiki.

Frecuencia de temas editados y de ediciones (por grupo y participantes)

\section{CONCLUSIONES}

En este trabajo exploratorio nos propusimos analizar el proceso de escritura colaborativa en línea llevado a cabo por estudiantes universitarios en el marco de un trabajo final a manera de ensayo, el uso que los participantes realizaron de los diferentes recursos para soportar su proceso de colaboración.

En primer lugar nos referiremos a las oportunidades que el proceso de escritura en línea ofrece para hacer un seguimiento pormenorizado de lo que los grupos hacen durante su proceso de trabajo. Como planteamos en nuestros apartados teóricos, uno de los aspectos potencialmente más relevantes del seguimiento es la oportunidad de identificar qué, quién, cómo, etc., hace o dice respecto del trabajo en grupo. Desde la perspectiva de Coll y colaboradores esto nos ofrece la posibilidad de transparentar, en el sentido de hacer visible para los profesores y/o para los participantes, aspectos de la colaboración que normalmente permanecen "opacos" y que no permiten, por un lado, la entrega de ayudas ajustadas al proceso del grupo y, por otro, la identificación de roles, acciones o actitudes individuales que no favorecen el proceso de trabajo. En este sentido, gracias al seguimiento del proceso hemos podido identificar lo que 
siempre presuponemos del trabajo en grupo: la implicación desequilibrada en la tarea que incluye desde la falta de atención por parte de todos los miembros del grupo hacia el trabajo previo con los contenidos hasta la habitual concentración en uno de los miembros, normalmente el más implicado, para escribir y poner en papel (o en el sistema Wiki, en nuestro caso) lo que seguramente se ha dicho o hecho previamente, pero que no se escribe, revisa, corrige o mejora entre todos los participantes implicados. Tenemos evidencia, gracias al arreglo realizado en nuestro trabajo, de quién hace qué y quién no hace qué; de quiénes aprovechan los espacios interactivos en línea para discutir, negociar y mejorar lo que han escrito y de quiénes utilizan los mismos recursos para procesos meramente acumulativos. Nuestros resultados nos permiten concluir hasta aquí que los grupos que leen en su conjunto sus materiales, que se frecuentan como grupo en los espacios de comunicación destinados para ello y que distribuyen sus tareas de escritura de una forma temporal y personal un tanto homogénea (escribir a lo largo del proceso más que sólo al final o escribir y mejorar tanto los textos propios como los de los otros) son los equipos de escritura colaborativa que se acercan mejor a un producto más acabado. En este sentido, nuestra metodología deja pendiente, aún, el análisis y definición de ayudas ajustadas durante el proceso. Aunque el profesor de los grupos analizados en este trabajo hizo un seguimiento puntual de las lecturas de los participantes, les invitó vía sus retroalimentaciones a hacer uso de las ideas escritas en dichos textos y participó en algunos de los foros o chats de pequeño grupo, no hemos estudiado aún cómo se aprovecha la oportunidad de seguimiento del proceso de escritura colaborativa para ayudar a aprender.

En segundo lugar, nuestros datos son una evidencia clara de lo que Storch (2005) propone que se ha de potenciar al estudiar la escritura colaborativa como proceso: que los estudiantes participen a lo largo de todo el proceso de escritura compartiendo la responsabilidad en la producción de un texto tanto a nivel de estructura como de contenido y de lenguaje. Nuestro análisis se ha quedado a un nivel meramente descriptivo, tendrá que pasar, mediante los instrumentos científicos pertinentes (análisis de contenido, etc.) a un nivel mucho más explicativo. No obstante, en este primer nivel, podemos indicar que los grupos que comparten la responsabilidad a lo largo de todo el desarrollo del texto y sus componentes son los grupos que mejor desarrollan su tarea de escritura colaborativa. Aún debemos contestar a preguntas cómo: ¿son mejores sus textos? ¿La evidencia que sobre el aprendizaje ofrecen sus textos es adecuada? ¿Hay diferencias en los productos textuales de los grupos que encontramos como más colaborativos, estratégicos -a los que les asignaríamos un sentido de verdadera co-autoría- y los que no lo fueron? 
En tercer lugar, recuperamos uno de los elementos que también proponían varios de los autores revisados en nuestra aproximación al tema y que ya planteamos en una de las preguntas abiertas anteriormente, nos referimos a la noción de co-autoría. Esta noción es la que, en un proceso de escritura colaborativa en línea, nos permitiría favorecer la aparición de mecanismos argumentativos tal y como Scardamalia y Bereiter (2003) suponen que deberían aparecer en una comunidad de indagación. En nuestro caso, tenemos evidencia, gracias al proceso de seguimiento de la escritura en línea, que al menos dos grupos, dada su participación distribuida en los temas del texto y sus mutuas revisiones, generaron una participación que nos permite concretizar la idea de co-autoría. Además, en su proceso aparece una combinación de compromiso, comunicación, gestión y acción muy relevante: todos los miembros leen todos los materiales que les permiten representarse la tarea, todos los miembros leen todos los contenidos, todos los miembros participan más o menos en el espacio de comunicación síncrona o asíncrona y, finalmente, todos los miembros participan en la aportación inicial o modificación posterior de todos los temas que conforman la estructura de su texto. La descripción de este proceso es lo que, desde nuestra perspectiva, operacionaliza la noción de co-autoría. Queda pendiente identificar cuáles son los elementos o aspectos que favorecen que los grupos aborden la tarea desde una perspectiva de co-autoría que permita la constitución de verdaderos grupos colaborativos de indagación. Sabemos que la metodología empleada en nuestra investigación orienta a los estudiantes hacía ese punto, pero también sabemos que puede deberse a la conformación misma de los grupos (que hicieron los estudiantes de acuerdo a sus criterios), a las características individuales de los participantes, a los roles asumidos por cada uno de ellos, etc.

En cuarto y último lugar, queremos destacar que la tecnología wiki, aunque aún poco amigable y comprensiva, favorece el seguimiento del proceso de escritura colaborativa en línea. Permite que los participantes gestionen sus procesos de escritura y que los profesores puedan hacer un seguimiento pormenorizado. Sin embargo, en esta primera aproximación no hemos podido evaluar los mecanismos que la tecnología ofrece para identificar con más precisión los mecanismos de gestión seguidos por los participantes: cellos mismos consultan las revisiones de sus pares? ¿Ellos mismos leen con detalle las versiones de unos y otros y, a partir de dicha relectura, generan oportunidades para la reflexión?

Finalmente, como ya se planteaba en la revisión teórica de nuestro objeto de estudio, quedan para el debate al respecto del estudio de la escritura colaborativa en línea no sólo la necesidad de investigar tanto los productos como los procesos que los estudiantes ponen en marcha cuando escriben colaborativamente, también quedan abiertas preguntas relativas a sus expectativas, sus actitudes y sus reflexiones, pero 
sobre todo, se abre el camino para la investigación sobre la forma en que la escritura colaborativa en línea puede aparecer como espacio de medicación entre lo que los profesores y los estudiantes, y los estudiantes entre sí, hacen en los espacios de enseñanza y aprendizaje universitario. Además de que quedan abiertas preguntas sobre la combinación de recursos tecnológicos para soportar el proceso y, sobre todo, sobre las características de los mismos dispositivos para ayudar en la gestión compartida de tareas abiertas como lo es la de escribir colaborativamente. ${ }^{4}$

\section{NOTAS}

1 Este artículo es la continuación del trabajo previo "Escritura colaborativa en línea. Un estudio preliminar orientado al proceso" que se presentó como comunicación en el Congreso Internacional Virtual Educa Brasil 2007.

2 El autor agradece al Dr. Felipe Tirado y a Alejandro Miranda, colegas de la UNAM FES-Iztacala, quienes participaron junto con el autor en el diseño de la secuencia didáctica y la recogida de datos que fueron la base para el trabajo preliminar presentado conjuntamente en el congreso antes citado.

3 Para mayor detalle véanse algunas experiencias aplicadas como las del proyecto Collaborate! (Collaborative Writing and Research in Higher education (http:// www.stanford.edu/group/collaborate/). Así como la gran diversidad de proyectos universitarios identificados como "Writing Center", por ejemplo: el MIT Online Writing and Communication Center (http://web.mit.edu/writing/) o el proyecto de investigación "Scaffolded Writing and Reviewing in the Disciplin" (http://www.lrdc. pitt.edu/schunn/SWoRD/index.html)

4 El autor desea extender un reconocimiento explícito y público al trabajo profundo, serio y de intenso valor formativo que caracteriza el proceso de evaluación externa "doble ciego" que la revista ha logrado instrumentar en su actual etapa.

\section{REFERENCIAS BIBLIOGRÁFICAS}

Barile, A.; Durso, F. (2002). Computermediated communication in collaborative writing. Computersin HumanBehaviour, 18, (173-190).

Carlino, P. (2005). Escribir, leer y aprender en la universidad. Una introducción a la alfabetización académica. Buenos Aires: FDCE.

Castells, M. (2006). La sociedad red: una visión global. Madrid: Alianza.

Chartier, R. (2000). Muerte o transfiguración del lector. $26^{\circ}$ Congreso de la Unión Internacional de Editores Aires. Buenos Aires. [en línea] Disponible en: http:// jamillan.com/para char.htm [consulta 2009, 18 de Mayo]

Coll, C. (2001). Constructivismo y educación: la concepción constructivista de la enseñanza y del aprendizaje. En Coll, C.; Palacios, J.; Marchesi, A. (Comps.). Desarrollo psicológico y educación. Vol. II, (157-188). Psicología de la Educación Escolar. Madrid: Alianza.

Coll, C. (2005). Lectura en la sociedad de la información. UOC papers, 1. [en línea] Disponible en: http://www.uoc.edu/ uocpapers/1/dt/esp/coll.pdf [consulta 2009, 18 de Mayo] 
Coll, C.; Martí, E. (2001). La educación escolar ante las nuevas tecnologías de la información y la comunicación. En: Coll, C.; Palacios, J.; Marchesi, A. (Comps.). Desarrollo psicológico y educación. Vol. II, (623-651). Psicología de la educación escolar. Madrid: Alianza.

Coll, C.; Mauri, T.; Onrubia, J. (2006). Análisis y resolución de casos-problema mediante el aprendizaje colaborativo. Revista de Universidad y Sociedad del Conocimiento. Vol. 3 (2).

Edwards, D.; Mercer, N. (1988). El conocimiento compartido: el desarrollo de la comprensión en el aula. Madrid: Paidós.

Erkens, G.; Jaspers, E.; Prangsma, M.; Kanselaar, G. (2005). Coordination processes in computer supported collaborative writing. Computers in Human Behavior, 18, (173-190).

Galegher, J.; Kraut, R. (1996). Computermediated communication for intellectual teamwork. En: Rada, R. (Ed.). Groupware and authoring. London: Academic Press.

Hayes, J. R.; Flower, L. S. (1986). Writing research and the writer. American Psychologist, 41, (1106-1113).

Keys, C. W. (1994). The development of scientific reasoning skills in conjuntion with collaborative assessments. An interpretative study of 6-9th grade students. Journal of Research in Science Teaching, 3, 9, (1003-1002).

Lévy, P. (2007). Cibercultura: la cultura de la sociedad digital. México: AnthroposUniversidad Autónoma Metropolitana.

Mercer, N. (2001). Palabras y mentes. Cómo usamos el lenguaje para pensar juntos. Barcelona: Paidós.

Miras, M. (2000). La escritura reflexiva. Aprender a escribir y aprender acerca de lo que se escribe. Infancia y Aprendizaje, $89,(65-80)$.

Olson, D. R. (1998). El mundo sobre el papel. El impacto de la escritura y la lectura en la estructura del conocimiento. Barcelona: Gedisa.

Onrubia, J.; Bustos, A.; Engel, A.; Segués, T. (2006). Usos de una herramienta de comunicación asíncrona para la innovación docente en contextos universitarios. Barcelona: IV Congreso Internacional de Docencia Universitaria $i$ Innovació. [en línea] Disponible en: http://www.psyed.edu.es/prodGrintie/ comunic/JO AB AE TS CIDUI o6.pdf [consulta 2009, 18 de Mayo]

Onrubia, J.; Coll, C.; Bustos, A.; Engel, A. (2006). Del diseño tecnopedagógico y el análisis de la práctica educativa al desarrollo tecnológico: retos para la mejora de Moodle. Tarragona: $V$ Congreso MoodleMoot'o6. [en línea] Disponible en: http://www.psyed.edu. es/prodGrintie/comunic/ JO CC AB AE Moodle 06.pdf [consulta 2009, 18 de Mayo]

Piscitelli, A. (2005). Distribución de contenidos y escritura colaborativa. En: Internet. La imprenta del siglo XXI. Cap. 5. Barcelona: Gedisa.

Rada, R.; Wang, W. (1997). Computersupported collaborative writing phases. Journal of Educational Technology Systems, 26, (137-149).

Scardamalia, M.; Bereiter, C. (2003). Knowledge Building. En: Guthrie, J. W. (Ed.).Encyclopedia of Education. Second Edition. New York, USA: Macmillan Reference. [en línea] Disponible en: $\quad$ http://ikit.org/fulltext/2003 knowledge building.pdf [consulta 2009, 18 de Mayo]

Schmidt, K. (2001). Computer-supported cooperative work and learning. Barcelona: UOC. FREREF ICT workshop. [en línea] Disponible en: http://www.uoc. edu/web/esp/art/uoc/schmidto202/ schmidt0202.html [consulta 2009, $18 \mathrm{de}$ Mayo] 
Stein, L.; Bernas, S.; Calicchia, D. (1997). Conflict talk: understanding and resolving arguments. En Giron, T. (Ed.). Conversation: Cognitive, communicative, and social perspectives. Typological studies in language. Vol. 34, (233-267). Amsterdam, The Netherlands: John Benjamins.

Storch, N. (2002). Patterns of interaction in ESL pair work. Language Learning, 52, $1,(119-158)$.

Storch, N. (2003). Relationships formed in dyadic interaction and opportunity for learning. International Journal of
Educational Research, 37, 3/4, (305322).

Storch, N. (2005). Collaborative writing: Products, process, and students' reflections. Journal of Second Language Writing, 14, (153-173).

Tedesco, J. C. (2000). Educar en la sociedad del conocimiento. Buenos Aires: FCE.

Wertsch, J. V. (1988). Vygostsky y la formación social de la mente. Buenos Aires: Aique.

\section{PERFIL ACADÉMICO Y PROFESIONAL DEL AUTOR}

Alfonso Bustos Sánchez. Candidato a Doctor en Psicología de la Educación por la Universidad de Barcelona. Miembro del Grupo de Investigación de Interacción e Influencia Educativa. Ha sido Director de Medios Educativos del INITE-México y Director del Centro de Alta Tecnología de Educación a Distancia-UNAM. Sus intereses de investigación se centran en temas relativos a los usos de TIC en educación superior, la enseñanza y el aprendizaje en redes asíncronas de aprendizaje y el análisis de procesos interactivos.

E-mail: abustos@ub.edu

DIRECCIÓN DEL AUTOR:

Universidad de Barcelona Facultad de PsicologíaDepartamento de Psicología Evolutiva y de la Educación, Campus Mundet Pg.Vall d'Hebron, 171 Barcelona, 08035

Fecha de recepción del artículo: 09/03/09

Fecha de aceptación del artículo: 18/05/09 